petition and at it's end, strict adherence to the draw, respect for the judges and a number of other rules.

As for the relationship between the teacher and the student, the first task of the teacher should be taking care of health, safety and well-being of the student. The teacher is obliged to promote accustoming future doctors with the help of acquired sport experience to regular physical activity for health strengthening, what will be a good example for patients.

In addition, deontological principles also determine certain requirements for the appearance of sportsman. Sportswear must correspond to the requirements, which is set by the specific of classes and by the rules of competitions in various sports. It is necessary to use replaceable shoes. The hair should be carefully braided, nails should be short-cropped. It is very important to use perfume cautious and moderately because some students can be intolerant of various substances, which can cause different reaction: hives, asthma attack and other impairments.

These deontological principles help to develope among students the right habits in terms of hygiene and sanitation.

In conclusion, it should be noted that such discipline as physical culture plays an irreplaceable role in the formation of ethically and deontologically correct standards, it can be an excellent foundation for the emergence of literate specialists in any branch of medicine.

\title{
UDC 616-092.9 DOI 10.22448/AMJ.2017.3.139-140 EFFICIENCY OF THE USE OF DIGIDROQUERCETIN IN GENERAL COOLING OF THE ORGANISM OF OLD RATS
}

\section{Namakonova V.S.}

\section{Amur State Medical Academy, Blagoveshchensk, Russian Federation}

Abstract. An analysis of the data obtained showed that the effect of low temperature on the organism of old rats leads to a significant decrease in the regenerative potential of the airway epithelium and the activation of lipid peroxidation (LPO) reactions in lung tissue and peripheral blood. The use of dihydroquercetin (DKV) on the background of cooling normalizes the reactions of LPO, activates proliferative processes in the epithelium of the respiratory system.

Key words: airway epithelium, low temperatures, reactions of lipid peroxidation, dihydroquercetin.

Itisknownthatlowambienttemperatureshavea negativeimpactontherespiratorysystem, whilethelevelsofLPOproducts increase reliably. Excess formation of these products can cause damage and death of cells. The reaction of cells and tissues to low temperatures depends on the age of the organism, because as the aging process, the level of metabolic processes decreases, which leads to excessive accumulation of LPO products and activation of oxidative stress [2]. In this connection, the study of a natural preparation of dihydroquercetin (DKV), which has a high antioxidant activity, is of special interest.

Materials and methods The work was performed on non-native white rats in the amount of 70, at the age of 19-20 months (old). The experimental animals were divided into groups: 1 . Intact; 2 . Animals that were subjected to general cooling for 14 days for 3 hours daily at a temperature of $-15^{\circ} \mathrm{C}$. 3. The animals of this group were orally injected with DHQ at a dose of 5 $\mathrm{mg} / 100 \mathrm{~g}$ for 2 weeks, then these rats were cooled for 14 days 3 hours daily at $-15^{\circ} \mathrm{C}$, continuing oral administration of DKB.

The object of the study was the cranial and caudal sections of the mucous membrane of the trachea, as well as the wall of the lobar and intralobular bronchi. For the study, pieces of tissue were cut from the appropriate sections. Half-thin sections were stained with toluidine blue. For a general morphological study, the preparations were stained with hematoxylin and eosin. Photographing was carried out on a microscope "Primo Star" Germany. Biochemical examination was performed on lung tissue and peripheral blood. To determine the products of lipid peroxidation and vitamin E, lipids from lung tissues were extracted by the Blay-Dyer method (1975). In the extract, diene conjugates, lipid hydroperoxides, malonic dialdehyde, vitamin $\mathrm{E}$ and ceruloplasmin were determined.

Results and discussion The internal lining of the mucous membrane of the proximal parts of the respiratory tract is represented by a multi-row prismatic ciliated epithelium located on the basal membrane. In old rats ciliated cells are often found in the process of elimination, both without morphological changes, and with signs of partial destruction of the ciliary apparatus and structural rearrangement of the nucleus [2]. With the action of cold from the epithelium, elements on the apical surface of which there are no cilia are often eliminated. There are zones of hypertrophic ciliated cells, as well as degenerate cells "bright cells" with signs of cytoplasm and nucleus dystrophy. The number of basal cells decreases significantly. In the intralobular bronchi, ciliated cells and Clara cells (CK) are present in the epithelium, capable of self-renewing their own population and differentiation into ciliate cells. Vaccination of the cytoplasm in some ciliated cells in old rats, part of the QC loses contact with the basal membrane. With a general cooling in old rats, the number of ciliate cells decreases by almost $20 \%$, many of them have degenerately altered nuclei, a vacuolarized cytoplasm. The effect of low temperatures leads to a significant increase in the level of LPO in the lung tissue; the indicators of diene conjugates are increased by $24.6 \%$, hydroperoxide by $12.4 \%$. The level of vitamin E, responsible for antioxidant protection, decreased by $44 \%$. A similar trend was also observed on the part of the blood indices, the content of diene conjugates increased sharply by $25 \%$, hydropericids by $29 \%$, malonic dialdehyde by $33 \%$, while a decrease in vitamin E by $13 \%$, ceruloplasmin by $17 \%$ was observed.

The use of DKV on the background of the effects of cold leads to a moderate increase in the number of basal cells. The ciliated cells acquire a more typical structure. In the small bronchus the epithelium is predominantly two-rowed, the number 
of Clara cells decreases, and if the bond of the $C C$ with the epithelial layer is weakly expressed upon cooling, Clark cells are in contact with the epithelium under the action of DKV. DKV has a normalizing effect on LPO reactions. In lung tissue, this results in a $16 \%$ decrease in diene conjugate values, hydroperoxides by $10 \%$ and vitamin E growth by $15 \%$. In peripheral blood, hydropericidia are significantly reduced by $20 \%$, MDA by $40 \%$, vitamin E increases by $13 \%$ and ceruloplasmin by $15 \%$.

With the aging of the body, the regeneration potential is reduced as a result of both a decrease in the number of stem cells, as well as the accumulation of metabolic products, which have a damaging effect on cells. With age, there are changes in connective tissue interactions in the epithelial lining of the respiratory tract, which leads to the development of involutive processes [1]. The effect of low temperature in older animals changes the level of proliferative processes more significantly, whichcandistortthedirection ofdifferentiation intheepithelium oftherespiratorysystem [3].Theresultsofthestudyindicate that the negative effect of low temperatures on the body can be reduced by using an antioxidant drug - dihydroquercetin.

\title{
References:
}

1. Pavlov, A.V. Histophysiology of the tracheal epithelium in rats in postnatal ontogenesis / A.V. Pavlov, L.I. Esev // Morphology. 2014. - T.146, №6. - P. 80-86.

2. Teselkin, Yu.O. Antioxidant effect of dihydroquercetin / Yu.O. Teselkin, B.A. Shambilova, I.V. Babenkova, G.I. Klebanov, N.A. Tyukavkin // Biophysics. - 1996. - T. 41, №3. - P. 620-624.

3. Celuyko, S.S. Modern views on the proliferation and differentiation of stem cells of the respiratory system in normal and cold exposure / S.S. Tseluyko, N.P. Krasavina, D.A. Semenov // Bulletin of the physiology and pathology of respiration. - 2012. - №45. P. 98-103.

UDK 617-089:616-08-031.81 DOI 10.22448/AMJ.2017.3.140-141

\section{POSSIBILITIES OF THERAPY OPTIMIZATION OF PATIENTS WITH SUPERFICIAL BURNING WOUNDS}

\author{
O.S. Olifirova, A.A. Kozka, E.A. Volosenkova
}

Amur State Medical Academy,

Gorkogo street, 95, Blagoveshchensk, 675000, tel. 8-(4162)-31-90-20, e-mail: agma@nm.ru

Abstract The analysis of treatment of 88 patients with burns of the I-II degrees (MKB-10) was carried out. Antioxidants (("Lavitol cosmetic", BAA "Lavitol-B") and hyperbaric oxygenation were applied in the complex treatment of patients of the main group (44) until the wound was completely epithelialized. The comparative assessment of results was carried out in a group of patients (44) who received traditional treatment. Antioxidants and hyperbaric oxygenation favorably affect the course of the wound process.The terms of spontaneous epithelization of the wound and inpatient treatment in patients of the main group decreased due to stimulation of the wound process.

Key words: superficial burns, dihydroquercetin, hyperbaric oxygenation, wound process.

Topicality. Burns therapy presents a global, social and economic problem in combustiology and surgery. It is known that among victims patients with superficial and border-line burns prevail making up $80 \%$ [1]. Such injuries do not lead to the development of a burn disease, they need the effective conservative treatment which can stimulate regenerative processes in a zone of the burn. According to modern literature hyperbaric oxygenation stirs up phagocytic activity of cells reparation and rate of their passing into the wound, stimulates action of antibacterial therapy [2]. Besides, in pathogenesis of a process developing in the wound great attention is paid to the active part of uncontrolled formation of free radicals in cells which further deceleration of reparation processes [3]. Application of antioxidants is pathogenetically substantiated. Because dihydroquercetin is a strong exogenous antioxidant having capillary protective, anti-inflammatory and moisture keeping effects then the probability of influence on the processes of LPO (lipid peroxidation) is high that intensifies the fibroblast reaction [4]. Objective: to assess the results of a complex application of hyperbaric oxygenation and dihydroquercetin ("Lavitol cosmetic", BAA "Lavitol-B") in the treatment of patients with superficial burns.

Materials and methods: the analysis of therapy results of 88 patients with burns of the I-II degrees (MKB-10) was conducted. Among them there were 49 men $(55,7 \%)$ and 39 women $(44,3 \%)$ at the age of 20-71 years. Etiology of thermal injuries: scalds - in $50(56,8 \%)$, flame burns - in $25(28,4 \%)$, contact burns - in $11(12,5 \%)$, scalds - in $2(2,3 \%)$ cases. Burn shock of the I-II degrees was observed in 6 patients with a burn area of $15 \%$ and $18 \%$. A burn disease was developing from 14 days till 2 months. On the average the area of thermal injuries made up 630,8 $\pm 6,7 \mathrm{~cm} 2$.

The main group (MG) included 44 patients receiving $\mathrm{HBO}$ and antioxidant therapy with bioflavonoid dihydroquercetin ("Lavitol cosmetic", BAA "Lavitol - B"). Therapy methods of patients of the main group were the following: after thorough cleansing of a burning wound, removing exfoliated epidermis and treatment with antiseptic ( $0,5 \%$ solution of chlorhexidine bigluconate) dressings with the powder of dihydroquercetin "Lavitol cosmetic" were performed in a day during 10-20 days. It was applied to the wound with the presence of microbes not more than $103-4$ b.m. per $1 \mathrm{~cm} 2$ in the layer of 1-2 mm. Atraumatic covering "Parapran" was applied over. At the same time hyperbaric oxygenation of 1,5-1,8 atmospheres during 40 minutes was conducted daily for 10 days. Besides, peroral intake of biologically active additive "Lavitol - B" was administered ( 1 capsule twice a day during meals daily for 15 days). Biologically active additive "Lavitol - B" contains natural antioxidant dihydroquercetin and polysaccharide arabinogalactan (1:3). Patients of the main group and the control group are similar in sex, age, degree and burns area.

Complex of therapy methods for patients from both groups included antibacterial and symptomatic treatment. 